\title{
Photoacoustic flow cytometry monitors cells circulating in vivo
}

\author{
Vladimir Zharov, Ekaterina Galanzha, Evgeny Shashkov, \\ Nicolai Khlebtsov, and Valery Tuchin
}

Real-time detection of nanoparticles and cells, including cancer, is now possible in blood and lymph flow.

Flow cytometry (FC) is a well-established technique that has revolutionized cell diagnostics in vitro. ${ }^{1}$ Extracted cells flow through an area irradiated by laser beams, and detectors record fluorescent and/or scattered light from these cells. Despite itslong history and many successful applications, conventionalFC is limited by theinvasive nature of cell extraction from a living organism, which may introduce artifacts. It also makes impossible long-term monitoring of the cells in their complex natural environment for studies of metastasis, cancer recurrence, immune function, or cell death. Furthermore, the cytotoxicity of the fluorescent labels used in most FC systems must be overcome before they can be used in humans. ${ }^{1-4}$

The main goal of our work has been to overcome these problems by developing a photoacoustic (PA) technique using laser radiation in the visible and near-IR for label-free detection of circulating cells in both blood and lymph flows. ${ }^{4-6}$ This technology also demonstrated a potentially nontoxic method of cell labeling using self-assembled nanoclusters ${ }^{7}$ of gold nanorods, carbon nanotubes, and other photothermal (PT) contrast agents with strong near-IR absorption and efficient transformation of laser energy into heat and accompanying effects.

The underlying principle of photoacoustic flow cytometry (PAFC) is the irradiation of individual cells in blood and lymph flow with one or a few focused laser beams operating at different wavelengths, then using an ultrasound transducer attached to the skin to record laser-induced acoustic waves (see Figure 1). We first performed a feasibility study in vitro, ${ }^{4}$ and then in vivo with a nude-mouse-ear model with well-developed clearly distinguishable blood vessels. We used an optical parametric oscillator with nanosecond pulses and a tunable wavelength (415-2300nm) to generate PA signals from single cells. We operated the light source mostly in the near-IR range $(700-920 \mathrm{~nm})$ because it offers maximum penetration of light into most biotissues enhanced with an optical clearing agent (see Figure 2).

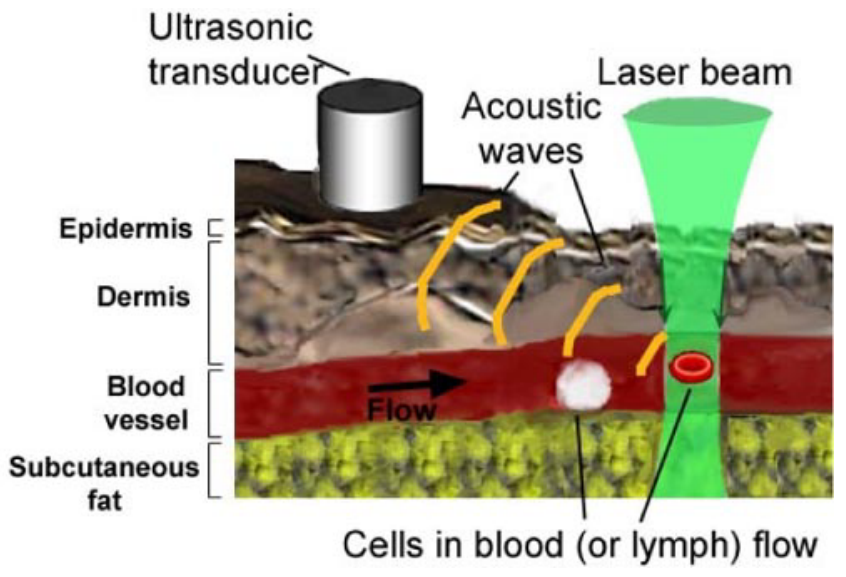

Figure 1. An ultrasonic transducer on the skin surface detects acoustic waves generated by a laser beam interacting with a cell or a few cells.

To verify reliability and make this new system more universal, we integrated the PAFC system with the following: two-beam PT flow cytometry in vivo, ${ }^{3,4}$ fluorescence image cytometry, ${ }^{4}$ which complements PAFC; and both light-scattering and speckle-flow cytometry. ${ }^{8}$ This integrated system provides label-free highlysensitive selective spectral detection of individual cells without causing photo-induced damage. It can sense cells with different natural absorption properties, including erythrocytes, leukocytes, and cancer cells such as leukemia and melanoma. Integrated signals are also highly sensitive to the cellular functional state (e.g., apoptosis or necrosis) and to the cellular response to environmental and therapeutic interventions (e.g., drugs or radiation). PAFC was also applied for routine monitoring of circulating nanoparticles (gold nanorods, nanoshells, carbon nanotubes), dyes (indocyanine green), and cells labeled with conventional dyes and gold nanorods that have a maximum absorption around $840 \mathrm{~nm}$. At this wavelength, the significant contrast between PA signals from cancer cells labeled with nanorods compared to PA signals from surrounding erythrocytes in blood flow allowed us to study the survival rate of circulating can-

Continued on next page 
a

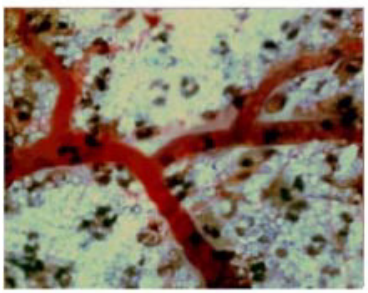

c

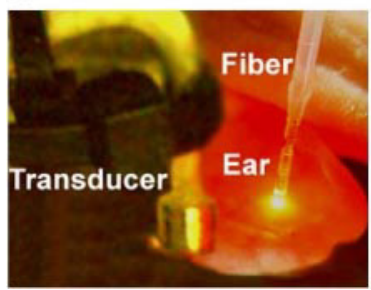

b

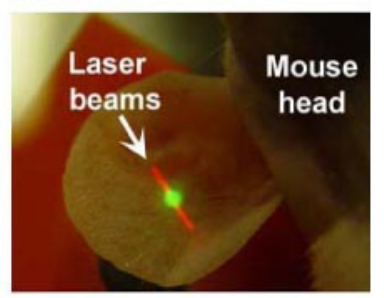

d

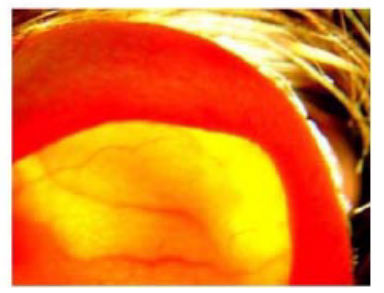

Figure 2. (a) A transmission image of part of a mouse ear after a clearing agent was applied. (b) The circular pump (green) laser beam is positioned in the middle of the linear probe (red) on the mouse ear. (c) The compact acoustic transducer is positioned near where a fiber delivers the laser radiation to the mouse ear. (d) In a human ear, blood vessels can be seen with white light.

cer cells in different functional states. For example, the half-life in circulation of live, dead, and apoptotic squamous carcinoma cells ranged from $1-1.5 \mathrm{~h}$, a few minutes, and 10-20min, respectively (see Figure 3).

Integrated flow cytometry with several channels provides the unique ability to rapidly detect cells and nanoparticles simultaneously in different blood and lymph vessels. It also allows detection of their accumulation in lymph nodes, the liver, and tumors. Furthermore, if we target cells with two sizes of nanorods, with absorption maxima near 700nm and 900nm, we can use spectral selection to simultaneously monitor apoptotic and necrotic cells. This sort of monitoring is important for fundamental studies of cell death in vivo and for the early diagnosis of diseases (e.g., Alzheimer's), or disease prevention (e.g., heart attacks). PAFC will help fill an existing gap in our knowledge of lymphatic function by focusing on transport proteins and cells for lymph-related diseases and cancer metastases within one of the typical pathways. These include the pathway from blood microvessels to tissue interstitium to the initial lymphatic and then afferent lymphatic, from there to the regional lymph node and on to the postnodal lymphatics.

In general, these studies have demonstrated, for the first time, high-sensitivity, time-resolved, noninvasive, quantitative realtime PA diagnostics of circulating cancer cells. Other potential applications of PAFC in vivo include molecular imaging and PA spectroscopy of single cells, ${ }^{5,6}$ measurements of flow velocity, ${ }^{3}$

a

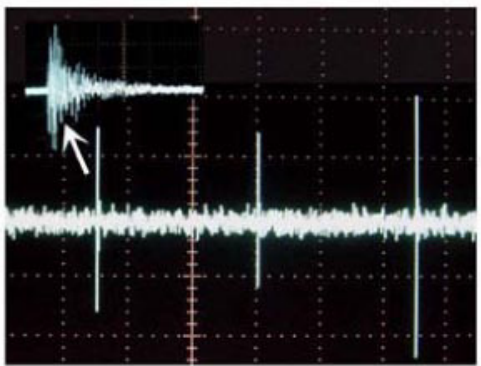

b

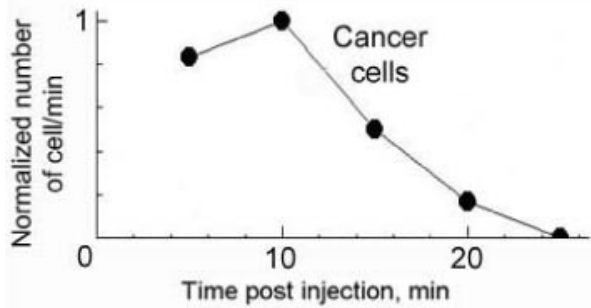

Figure 3. (a) Two traces of photoacoustic (PA) signals from individual erythrocytes in blood flow. The top trace shows an uncompressed signal with a time scale of $100 \mu \mathrm{s} / d i v$. The lower trace shows a timecompressed pulse, with a time scale of $40 \mathrm{~ms} / \mathrm{div}$. The 570-nm laser fired $8 n$ s pulses with energy density of $35 \mathrm{~mJ} / \mathrm{cm}^{2}$. (b) The contrast between normal and gold-nanorod-labeled cancer cells circulating in blood flow allow us to plot a mormalized number of cancer cells as a function of time after injection. To obtain this data, we used an $840 \mathrm{~nm}$ laser creating 8ns-long pulses with an energy density of $25 \mathrm{~mJ} / \mathrm{cm}^{2}$.

and supersensitive detection of individual cell response to lowdose radiation. ${ }^{4}$ This method could also be used to integrate noninvasive PA diagnostics with PT therapy of cancer cells: using PA triggering of therapeutic laser pulses to selectively kill only rare metastatic cancer cells labeled with nanorods without harming normal blood cells (because the difference in the photodamage threshold of these cells is more than two orders of magnitude in the near-IR range). ${ }^{6}$ PAFC could also be used for fundamental studies of blood oxygenation and aggregation at the single-cell level. ${ }^{6}$ This technique has great potential for use in humans, particularly for noninvasive monitoring of circulating cells in different vessels (e.g., in the retina, fingernails, or ears), or with a fiber chip-based catheter in vessels.

This work was funded in part by grants EB000873, EB001858, and EB0005123 from the US National Institutes of Health.

Continued on next page 


\section{Author Information}

\section{Vladimir Zharov, Ekaterina Galanzha, Evgeny Shashkov}

Philips Classic Laser Laboratories

University of Arkansas for Medical Sciences

Little Rock, AR

Vladimir Zharov is director of Philips Classic Laser Laboratories and both a professor and director of laser research at the University of Arkansas for Medical Sciences.

Ekaterina Galanzha is a visitor from Saratov State University, Russia.

Evgeny Shashkov is a visitor from Prokhorov General Physics Institute, Moscow, Russia.

\section{Nicolai Khlebtsov}

Laboratory of Nanoscale Biosensors

Institute of Biochemistry and Physiology of Plants and Microorganisms

Saratov, Russia

\section{Valery Tuchin}

International Institute of Optics and Biophotonics

Saratov State University

Saratov, Russia

\section{References}

1. A. L. Givan, Principles of flow cytometry: an overview, Methods Cell Biol. 63, pp. 19-56, 2001.

2. J. Novak, I. Georgakoudi, X. Wei, A. Prossin, and Lin CP, In vivo flow cytometer for real-time detection and quantification of circulating cells, Opt. Lett. 29, pp. 77-79, 2004

3. V. Zharov, E. Galanzha, and V. Tuchin, Photothermal imaging of moving cells in lymph and blood flow in vivo, Proc. SPIE 5320, pp. 256-263, 2004.

4. V. P. Zharov, E. I. Galanzha, and V. V. Tuchin, In vivo photothermal flow cytometry: Imaging and detection of cells in blood and lymph flow (invited review), J. Cell Biochem. 97, pp. 916-932, 2006.

5. S. I. Kudryashov, S. D. Allen, E. I. Galanzha, E. Galitovskaya, and V. P. Zharov, Photoacoustics of individual live cells and particles (invited review), Proc. SPIE 6086, pp. 143-154, 2006

6. V. Zharov, E. Galanzha, E. Shashkov, N. Khlebtsov, and V. Tuchin, In vivo integrated photoacoustic flow cytometry: Application for monitoring circulating cancer cells labeled with gold nanorods, Fifth Inter-Institute Workshop on Optical Diagnostic Imaging from Bench to Bedside at the National Institutes of Health, p. 126, September 2006. Modified version was submitted to Opt. Lett.

7. V. Zharov, J. W. Kim, M. Everts, and D. Curiel, Self-assembling nanoclusters in living systems: Application for photothermal nanodiagnostics and nanotherapy (invited review), J. Nanomed. 1, pp. 326-345, 2005.

8. V. Zharov, Y. Menyaev, E. Shashkov, E. Galanzha, B. Khlebtsov, A. Scheludko, D. Zimnyakov, and V. Tuchin, Fluctuation of probe beam in thermolens schematics as potential indicator of cell metabolism, apoptosis, necrosis and laser impact, Proc. SPIE 6085, pp. 10-21, 2006. 